\title{
High-throughput measurement of protein stability in microtiter plates.
}

Jean P. Aucamp, Ana M. Cosme, Gary J. Lye and Paul A. Dalby

The Advanced Centre for Biochemical Engineering, Department of Biochemical

Engineering, University College London, Torrington Place, London, WC1E 7JE, UK

\section{Corresponding author:}

Dr. Paul Dalby

Dept of Biochemical Engineering

University College London

Torrington Place, London WC1E 7JE, UK

Tel: +44 207679 2962;

Fax: +442073832348

p.dalby@ucl.ac.uk<mailto:p.dalby@ucl.ac.uk>

Short running title:

High-throughput measurement of protein stability 


\section{Summary}

The direct determination of protein stability at high throughput has applications in proteomics, directed evolution, and formulation. Each application places different requirements on the accuracy of stability or transition mid-point determination. The measurement of protein stability by chemical denaturation has been previously performed at medium throughput and high accuracy using autotitrating fluorometers, after removing proteins from the 96-well plate format in which they were expressed and purified. Here we present a higher-throughput method for measuring and indexing the stability of proteins maintained within the 96 -well format using a fluorescence microplate reader. Protein unfolding transitions were monitored by tryptophan fluorescence at $340 \mathrm{~nm}$, and assessed using bovine and equine cytochrome $c$ (cyt $c$ ), as well as bovine serum albumin (BSA) stabilised with various amounts of palmitic acid. Two different approaches for generating unfolding curves in microtiter plates have been evaluated for their accuracy and applicability.

Unfolding curves generated by the serial addition of denaturant into single wells, allowed high-throughput stability screens capable of identifying protein variants with unfolding mid-point differences of $0.15 \mathrm{M}$ denaturant concentration or larger. Such a method would be suitable for screening large numbers of proteins, as typically generated for directed evolution. Unfolding curves generated using one well per denaturant concentration allowed for medium-throughput stability screening and generated more accurate and precise stability values $\left(C_{1 / 2} \pm 0.05 \mathrm{M}, m_{\mathrm{G}}\right.$ and $\left.\Delta G_{\mathrm{H}_{2} \mathrm{O}}\right)$ for cyt $c$ that are similar to values reported in literature. This method is suitable for screening the smaller numbers of proteins generated in proteomic research programmes. By using BSA stabilised with various palmitate concentrations, and 
simple numerical indexing, it was shown that both experimental methods can successfully rank the order of protein stability.

\section{Keywords}

directed evolution, high-throughput screening, proteomics, formulation, protein stability, automation. 


\section{Introduction}

The measurement of protein stability is essential for elucidating protein function in vivo, engineering the improved stability of therapeutic proteins or biocatalytic enzymes, and formulating proteins for therapeutic delivery. For example, the biological function of proteins in vivo is dependent on their folded state and stability, and can change in response to signals that alter protein folding and assembly (Dyson and Wright, 2002). Disease conditions linked to protein misfolding and aggregation are also often associated with less stable protein variants (Dobson, 1999). While complete genome sequencing projects and proteomic tools can identify novel proteins and provide key information for understanding the complex processes of life (Yarmush and Jayaraman, 2002), the measurement of protein stability is an essential part of understanding these biological processes. The successful application of tagged-protein purification (Braun and LaBaer, 2003) and high-throughput automation will further enable the development of rapid screens for characterising the physicochemical properties of many purified proteins in parallel. Currently, however, major bottlenecks in the quest for rapid characterisation of thousands of potentially novel and as yet functionally unassigned genes, are frustrating our understanding of cellular protein networks and the advancement of bio-molecular medicine. The ability to rapidly characterise the biophysical properties, such as stability, of thousands of newly identified proteins would greatly enable our ability to predict protein function in vivo and to design new modes of action for therapeutics.

Advances in high-throughput protein engineering techniques have also placed high demands on the biophysical characterisation of large numbers of protein variants in the search for novel structure-function combinations. Such directed evolution 
techniques have been successfully used to improve the properties of therapeutic proteins (Kurtzman et al., 2001; Page et al., 1999) and enzymes for industrial applications (Dalby, 2003). The vast number of protein variants generated from directed evolution and proteomic drug target research programmes poses a great challenge for the high-throughput and accurate determination of protein properties such as activity, stability, structure (Heinemann et al., 2001) and ligand-binding affinity. The harsh conditions of industrial biocatalytic processes (Burton et al., 2002) frequently require enzymes that are stable and active at high temperatures (Demirjian et al., 2001) or in the presence of organic solvents (Schmid et al., 2001). The highthroughput determination of protein stability would enable stabilising mutations to be identified from large libraries of enzyme variants, under the same conditions used in biocatalysis. Similarly, therapeutic proteins require optimal formulation to improve shelf life and drug delivery (Cueto et al., 2003; Remmele, Jr. et al., 1998) and a highthroughput stability measurement would enable many combinations of excipients to be rapidly tested for their effect on protein stability.

High-throughput measurements of protein stability are often obtained indirectly by monitoring protein aggregation (Won et al., 1998) or residual activity after incubation at elevated temperatures (Giver et al., 1998). Both screens rely on irreversible inactivation of the protein upon unfolding, and also the assumption that positive variants result from resistance to denaturation under the test conditions. Although these indirect screens have been applied successfully, they may not easily distinguish differences in stability for proteins that spontaneously refold when returned to the activity-assay conditions.

A high-throughput screen that directly measures protein stability is preferred, particularly for directed evolution applications where it is well known that 'you get 
what you screen for' (Schmidt-Dannert and Arnold, 1999). For example, mutations may arise that promote reversible protein folding rather than resistance to unfolding at elevated temperatures. Recent advances in instrument technology currently allow the direct high-throughput measurement of protein stability by differential scanning calorimetry (Weber and Salemme, 2003) at 50 samples per day, or by monitoring the protein transition from native to unfolded state by means of $\mathrm{H} / \mathrm{D}$ exchange and MALDI-MS analysis (Ghaemmaghami et al., 2000) at 1000 samples per day.

The unfolding transition of proteins can also be observed by measuring their tryptophan fluorescence upon perturbation with a chemical denaturant or a temperature shift. The average fluorescence reflects the change in local environment around the tryptophan residues and provides a direct means of assessing protein unfolding (Lakowicz, 1999). The unfolding transition of most soluble proteins can be obtained using commercially available automated-titrating fluorometers. Instrumentation used for automated unfolding experiments must fulfil several criteria to ensure high-precision data is obtained (Schwehm and Stites, 1998). These include accurate and precise titration of solvent denaturants, low signal drift over the timecourse of the experiment and software that automates thermodynamic calculations based on the data obtained. High-precision studies have been conducted previously, for the analysis of a mutant library of the fast unfolding protein, eglin c, using a semiautomated titrating fluorometer (Edgell et al., 2003) at a throughput of approximately 64 samples per day. Recent work (Scheich et al., 2004) has also used the tryptophan fluorescence of protein solutions, as determined using a microplate reader, in conjunction with hydrophobic interaction chromatography analysis to evaluate whether a protein in solution is folded or not. However, this work only measured the total fluorescence at $340 \mathrm{~nm}$ for the solutions and did not attempt to denature the 
protein and determine their stability.

Here we describe a high-throughput unfolding procedure using a commercially available fluorescence plate reader with titrating syringe pump, that can be linked by automation to the high-throughput expression and purification of proteins as described previously (Edgell et al., 2003). The accuracy of the procedure was demonstrated by analysing the unfolding of oxidized bovine and equine heart cytochrome $c$ (cyt $c$ ), as well as bovine serum albumin (BSA) containing increasing concentrations of palmitic acid. The change in conformation of BSA upon stabilisation with palmitic acid was observed. The stability measurements for these proteins were compared with values reported in the literature, obtained under similar conditions.

\section{Materials and Methods}

\section{Sample preparation}

All chemicals and proteins were obtained from Sigma-Aldrich Company Ltd (Poole, UK), except guanidine hydrochloride (GdnHCl) (Perbio Sciences, Cheshire, UK). Fat free BSA, and cyt $c$ from bovine heart and equine heart were used without further purification. The buffers used throughout the study were $100 \mathrm{mM}$ Tris, $\mathrm{pH} 7.0$ for cyt $c$ and $60 \mathrm{mM}$ sodium phosphate, $\mathrm{pH} 7.0$ for BSA.

Cyt $c$ was oxidized by adding $20 \mu \mathrm{l}$ of $0.1 \mathrm{M}$ potassium ferricyanide per $1 \mathrm{ml}$ protein solution. The oxidised cyt $c$ preparations were dialyzed for $24 \mathrm{hr}$ at $4{ }^{\circ} \mathrm{C}$ with two buffer exchanges using $10 \mathrm{kDa}$ MWCO dialysis tubing (Perbio Science, Cheshire, UK). The absence of an absorbance peak at $550 \mathrm{~nm}$ confirms the complete oxidation of the heme moiety (Yonetani, 1965). Prepared cyt $c$ samples were used immediately. 
BSA samples containing various molar ratios of palmitic acid were prepared with the procedure of Brodersen et al., 1988. Briefly, $300 \mu \mathrm{M}$ BSA solution in ROwater was prepared and adjusted to $\mathrm{pH} 9$ with $\mathrm{NaOH}$. A $90 \mathrm{mM}$ ethanolic solution of palmitic acid was prepared and sufficient volume added to the BSA solution to give BSA:palmitate molar ratios from 1:1 to 1:6. The preparations were dried using a centrifugal vacuum evaporator (Speed-Vac SVC100H, Savant Instruments, NY) and the dried material stored at $-20^{\circ} \mathrm{C}$ until used.

The concentrations of protein stock solutions were determined from absorbance measurements, using the molar absorption coefficients values of 106000 $\mathrm{M}^{-1}$ at $410 \mathrm{~nm}$ for cyt $c$ (Margoliash and Frohwirth, 1959) and $43890 \mathrm{M}^{-1}$ at $280 \mathrm{~nm}$ for BSA (Pace et al., 1995). The denaturant stock buffers used were $100 \mathrm{mM}$ Tris, $\mathrm{pH}$ 7.0, 6.5 M GdnHCl for cyt $c$ and $60 \mathrm{mM}$ sodium phosphate, $\mathrm{pH}$ 7.0, 9.0 M urea for BSA.

\section{Stability measurement}

All protein unfolding transitions were measured in F-type, polystyrene, 96well microtiter plates (Greiner Bio-One Ltd, Gloucestershire, UK) using a fluorescence microplate reader, Fluostar Optima (BMG Labtechnologies Ltd, Bucks, UK), fitted with a syringe pump for automation of reagent injection. Protein unfolding was monitored by intrinsic tryptophan fluorescence using a $340 \pm 10 \mathrm{~nm}$ emission window filter with excitation at $280 \mathrm{~nm}$.

Two different protocols were used to generate protein-unfolding curves in microtiter plates, one in which the final volume at each denaturant concentration was fixed and the other in which serial addition of denaturant to a protein solution gradually increased the total volume. Both protocols were automated, using the 
syringe pump to inject all the solutions. The two approaches allowed us to compare the effect of decreasing protein concentration on stability determination with that of keeping it constant.

Fixed volume denaturation: $20 \mu \mathrm{g}$ cyt $c$ in a volume of $50 \mu \mathrm{l}$, or $40 \mu \mathrm{g}$ BSA in $20 \mu \mathrm{l}$ was loaded into each well of a 96-well microtiter plate. For cyt $c$, wells were filled with different amounts of buffer containing $0 \mathrm{M}$, and then $6.5 \mathrm{M} \mathrm{GdnHCl}$ to a final volume of $215 \mu \mathrm{l}$, to generate a final denaturant concentration range of 0 to 3.8 M. For BSA, wells were filled using buffer containing $0 \mathrm{M}$ and then $9 \mathrm{M}$ urea to a final volume of $280 \mu \mathrm{l}$, for a urea concentration range of $0 \mathrm{M}$ to $8.4 \mathrm{M}$. Each sample was repeated in triplicate.

Serial addition of denaturant: With this protocol, protein was loaded into each well followed by the addition of small volumes of buffered denaturant stock. Each addition was followed by 30 seconds of mixing by orbital shaking at $350 \mathrm{rpm}$, and 15 minutes of equilibration prior to taking measurements. Volumes for serial addition were adjusted to provide nearly equidistant data points on the unfolding curve. The minimum volume of the initial protein solution that can cover the well bottom is 50 $\mu 1$. Using this protocol, $20 \mu \mathrm{g}$ cyt $c$ or $40 \mu \mathrm{g}$ BSA was loaded per well.

\section{Auto-injector measurement}

Several $3 \mu 1$ and $5 \mu 1$ injections were made into a single well of a pre-weighed 96-well microtiter plate. The microtiter plate was weighed after each injection. Measurements were plotted as total volume injected against the total weight increase. In another test, larger volumes $(10,25$ and $50 \mu \mathrm{l})$ were injected using the same procedure. Weight measurements obtained were deemed reliable based on agreement 
with dispensed volumes and the density of water at $25^{\circ} \mathrm{C}$.

\section{Stability calculations}

All data fitting and analysis was performed using the software package SigmaPlot 8.0 (SPSS UK Ltd., Surrey, UK). For a two-state model the native and unfolded protein species are the only significant populations present. Pre- and postunfolding baselines are determined using [Eq. (1)] and [Eq. (2)] where $F^{\circ}{ }_{N}$ is the fluorescence signal for the native state at $0 \mathrm{M}$ denaturant, and $m_{\mathrm{N}}$ is the slope $\left(d F_{\mathrm{N}} / d\right.$ [D]) for the native state baseline. $F^{\circ}$ and $m_{\mathrm{U}}$ are the corresponding parameters for the unfolded state.

$F_{o b s, N}=F_{N}^{\circ}+m_{N}[D]$

$F_{o b s, U}=F_{U}^{\circ}+m_{U}[D]$

The intercept and slope values were substituted into [Eq. (3)] (Santoro and Bolen, 1988) where $R$ is the gas constant $\left(1.987 \mathrm{cal} \cdot \mathrm{mol}^{-1}\right)$ and $T$ the absolute temperature $(298 \mathrm{~K}) . C_{1 / 2}(\mathrm{M})$ is the denaturant concentration where half the protein is in the unfolded state and $m_{\mathrm{G}}\left(\mathrm{kcal} \cdot \mathrm{mol}^{-1} \cdot \mathrm{M}^{-1}\right)$ is the change in free energy of unfolding with respect to the denaturant concentration. The raw fluorescence data was fitted to [Eq. (3)] and the two parameters, $m_{\mathrm{G}}$ and $C_{1 / 2}$, estimated with errors. 


$$
F_{o b s}=\frac{\left(F^{\circ}{ }_{N}+m_{N}[D]\right)+e^{\left(\frac{m_{G}\left[D-C_{1 / 2}\right]}{R T}\right)}\left(F_{U}^{\circ}+m_{U}[D]\right)}{e^{\left(\frac{m_{G}\left[D-C_{1 / 2}\right]}{R T}\right)}+1}
$$

The free energy of protein unfolding was calculated by the linear extrapolation method (Pace, 1986), in which the free energy of unfolding is a function of denaturant concentration [Eq. (4)]. The $\Delta \mathrm{G}_{o b s}$ values were calculated from data points in the transition region using [Eq. (5)] and the relationship $\Delta \mathrm{G}_{o b s}=-\mathrm{RT} \ln \mathrm{K}_{o b s}$. The $\Delta \mathrm{G}_{o b s}$ values were plotted against denaturant concentrations and extrapolation towards $0 \mathrm{M}$ determines the stability of the native protein $\left(\Delta G_{H_{2} O}\right)$.

$\Delta G_{o b s}=\Delta G_{H_{2} O}+m_{G}[D]$

(4)

$K_{o b s}=\frac{F_{o b s}-F_{o b s, N}}{F_{o b s, U}-F_{o b s}}$ 


\section{Results and Discussion}

\section{Choice and relevance of model proteins}

Our aim was to develop and test the direct measurement of protein stability by tryptophan fluorescence in microtiter plates, using proteins with well-defined unfolding properties and previously reported stability values. Use of previously characterised protein models has permitted us to assess the relevance of absolute stabilities obtained by this method, compared to the measurement of changes in stability upon, for example, mutation or addition of excipients.

The first protein chosen, cyt $c$ has applications in industrial biocatalysis (Vazquez-Duhalt, 1999) and is known to unfold reversibly in the oxidised form (Xu et al., 1998). The ability to screen variant cyt $c$ libraries by fluorescence during unfolding is anticipated to allow the identification of mutations that extend the lifetime for this enzyme under biocatalytic process conditions. The need to identify differences in protein stability upon mutation is paramount in such directed evolution experiments, whereas the absolute determination of stability is not necessary.

Ligands, cofactors, metal ions, substrates and other excipients often confer extra stability upon binding to proteins. The thermodynamic stabilisation of BSA with fatty acid molecules is one such example (Ahmad and Qasim, 1995). BSA has three fatty acid binding sites, two of which are located in the C-terminal side, and binds up to 7 molecules of palmitate per protein (Hamilton et al., 1991; Reed, 1986). The palmitate molecules stabilise the C-terminal domains involved in binding and have a marked effect on the tolerance of BSA to denaturant induced unfolding. This second choice of model protein allows us to determine the suitability of microtiter plate stability measurements for elucidating the biochemical function of proteins, and 
also for formulating therapeutic proteins with optimal shelf-life.

\section{Accuracy of microwell scale unfolding measurements}

The determination of protein stability by equilibrium unfolding techniques requires that a large amount of measurements at different denaturant concentrations be made. The largest source of variation and experimental error originates from differences in the final protein concentration for each denaturant concentration solution. This can be eliminated by performing an unfolding experiment starting with a half-full cuvette, adding denaturant and correcting for the dilution effect on the signal if necessary (Schwehm and Stites, 1998). In doing so, accurate titration becomes the only major concern. This method was applied here in microwells and since the total fluorescence of each well was measured, dilution of the protein solution should have little effect on the signal. Care has to be taken to ensure that the working protein concentration produces fluorescence in the linear response range of the instrument. High protein concentrations show a large fluorescence drift with dilution, most likely due to the inner filter effect (Lakowicz, 1999). Fluorescence signal drifts of only $2.15 \%$ and $2.88 \%$ were observed respectively over a four-fold dilution range for a $0.6 \mathrm{mg} \cdot \mathrm{ml}^{-1} \mathrm{BSA}$ (Fig. 1) and a $0.25 \mathrm{mg} \cdot \mathrm{ml}^{-1}$ bovine $c y t c$ (data not shown) initial concentrations. Dilution effects on total fluorescence were therefore not corrected for in our calculations. Denaturant stock solutions were prepared at a concentration that permitted complete protein unfolding to be achieved without exceeding a four-fold dilution of the initial protein concentration. This requires some prior knowledge about the unfolding midpoints of proteins to be analysed, or a rough pre-screen of unfolding in microtiter plates using various stock solutions.

The accuracy of both large and small volume titration using the syringe pump 
as determined by the manufacturer was verified, as this was expected to have the greatest effect on the accuracy of the protein unfolding data. Water was auto-titrated into wells and the cumulative mass of water measured and plotted against the total volume dispensed (Fig. 2). The average slope calculated was very similar to the density of water $\left(0.997 \mathrm{~g} \cdot \mathrm{cm}^{-3}\right.$ at $\left.25^{\circ} \mathrm{C}\right)$, verifying the accuracy of titration (precision was not possible to determine for the low volumes dispensed). Linearity was observed down to $3 \mu$ volume injections, making this the minimum accurately dispenseable volume. This restricts the minimum denaturant concentration increase that could be made using serial addition. Incremental jumps in denaturant concentration were largest for the first injections, and the $3 \mu$ l volume injection minimum defined the number of useful data points that could be obtained for the initial baseline of the transition curve.

The equilibration time between injections of denaturant is critical to the accurate determination of protein stability. The time required may vary considerably between proteins and can, therefore, adversely affect the throughput at which measurements can be made. Consequently it is expected that for rapidly equilibrating proteins, the serial addition mode would provide the highest throughput, whereas for slowly equilibrating proteins, the fixed volume mode would be best. For unknown protein samples, a pre-screen of various equilibration times at a given denaturant concentration is, therefore, necessary to determine the the best experimental mode to use. The equilibration times were optimised for the serial addition mode, to ensure that cyt $c$ and BSA unfolding both reached equilibrium. It was determined empirically that 15 minutes between injections were sufficient for both cyt $c$ and BSA to fully equilibrate, resulting in serial addition experiments that took up to 10 hours for 25 denaturant concentrations. The equipment used produced a slight rise in temperature 
of $3{ }^{\circ} \mathrm{C}$ and approximately $5 \%(\mathrm{w} / \mathrm{w})$ evaporation over the duration of each experiment, highlighting potential improvements that could be made instrumentally.

Protein unfolding experiments were initially conducted in clear, and black plates to determine the type best suited for the application. Clear plates were found to give results comparable to values reported in the literature (Table I). The results reported here were all obtained using clear plates. All the wells had a uniform background signal, except for wells situated at the edge of the plate which had higher background signal. Only the inner wells were used for analysis with the fixed volume method to obtain reproducible replicates. The background noise was additive and constant for all readings taken from a single well, thus had no effect on results obtained by analysis with the serial addition method. This is exemplified by showing that an unfolding curve obtained from an edge well (Fig. 3B, dataset $(\boldsymbol{\Delta})$ ) has a similar profile to other unfolding curves obtained using inner wells. However, the $C_{1 / 2}$ obtained from the edge well $(2.51 \mathrm{M})$ was distinctly lower than those obtained for all other wells, ranging from $2.63-2.75 \mathrm{M}$, and thus only inner wells should be used for improved accuracy and precision. The clear plates had a higher background noise, and the difference between native and unfolded baseline fluorescence signals were 3-5 times lower, than for black plates (data not shown). However, black plates produced unexplained, and inconsistent, volume dependant baseline drifts that distorted the $\mathrm{m}$ values obtained with stability calculations.

\section{Conformational transition curves for cytochrome $c$}

Conformational transition curves for cyt $c$ from bovine and equine heart, obtained with the fixed volume method, are plotted as the average fluorescence intensity from triplicate measurements (Fig. 3A). Curves obtained with the serial 
addition method are plotted as nine replicates performed in parallel (Fig. 3B).

Stability values, $C_{1 / 2}, m_{\mathrm{G}}$-value (estimated with non-linear fitting) and $\Delta G$ (estimated with linear extrapolation) for both methods are summarised in Table I. The reported stability values for the fixed volume method are obtained from one analysis and for the serial addition method they are averages of estimates for each of the nine parallel stability analyses. The inter-run variability was calculated from three separate experiments conducted on separate days, using different stock and protein solutions (Table I).

$C_{1 / 2}$ estimations using the fixed volume method were accurate enough to rank protein variant stability in a population with similar $C_{1 / 2}$ values (typically $\pm 0.05 \mathrm{M}$ ). Estimations of $C_{1 / 2}$ and $m_{\mathrm{G}}$-values compare well with reported values in the literature (Table I). Mid-points of unfolding for proteins can be estimated to an accuracy of \pm $0.15 \mathrm{M}$ using the serial addition method, but values of $m_{\mathrm{G}}$ in this case are significantly overestimated. Data obtained with the serial-addition, high-throughput method estimates the $C_{1 / 2}$ value of equine cyt $c$ to be higher than that of the bovine counterpart. This is the opposite result as compared to the fixed volume and literature values where bovine cyt $c$ has the higher $C_{1 / 2}$ value $(2.46 \pm 0.05 \mathrm{M}$ and $2.51 \pm 0.19 \mathrm{M}$ for bovine and equine cyt $c$ respectively obtained from three separate runs of nine datasets each). However, the errors associated with $C_{1 / 2}$ values calculated by the serial addition method $( \pm 0.08-0.14 \mathrm{M})$, are of similar magnitude to, and may partly account for, this discrepancy. Larger variation in pre- and post-transition baselines as well as evaporation and the small increase in temperature over the longer experiment time for serial addition are the most likely reasons for this occurrence. The ability to rank protein stabilities in terms of $C_{1 / 2}$ is sufficient for directed evolution screens, where the key requirement is to find enzymes that resist unfolding under the 
conditions required in a bioreactor (Burton et al., 2002). It is also useful for therapeutic formulation in which a combination of excipients is desired that improve the resistance of the protein to unfolding (Remmele, Jr. et al., 1998).

The large error on $m_{\mathrm{G}}$-value calculation using the serial addition mode $(6.2 \pm$ $1.8 \mathrm{kcal} \cdot \mathrm{mol}^{-1} \cdot \mathrm{M}^{-1}$ for bovine cyt $c$ ) is a result of the inaccurate determination of the pre- and post-unfolding baselines. $K_{\text {obs }}$ values, also determined by extension of the stable baselines into the transition region, and the subsequent calculation of $\Delta G_{\text {obs }}$ values are also adversely affected. Moreover, data used for linear extrapolation estimates show average regression coefficients of $0.93 \pm 0.11(n=36)$ suggesting that the large variations in $\Delta G_{\mathrm{H}_{2} \mathrm{O}}$ values originate from erroneous baseline determination. In this mode the initial additions cause large increments in the denaturant concentration, thus limiting the number of data points obtainable in this region. This limitation is a direct result of the minimum volume that can be accurately titrated by the syringe pump and suggests a critical area for further improvement. This leads us to conclude that with present instrumentation, $\Delta G$ values cannot be determined accurately with the serial addition method using linear extrapolation. $\Delta G_{\mathrm{H}_{2} \mathrm{O}}$ values obtained with the fixed volume method are reasonably accurate, but the $\Delta \Delta G$ for bovine and equine cyt $c$ is underestimated using linear extrapolation $\left(0.27 \mathrm{kcal} \cdot \mathrm{mol}^{-1}\right.$ calculated compared to the literature value of $\left.1.08 \mathrm{kcal} \cdot \mathrm{mol}^{-1}\right)$.

\section{Conformational transition curves for BSA:palmitic acid mixtures}

The conformational transition curves of BSA generated with the fixed volume method required a $9 \mathrm{M}$ urea stock-solution to ensure complete unfolding of the most 
stable protein preparation. GdnHCl, a stronger denaturant (Pace, 1986), was used instead of urea for the serial addition unfolding analysis for two practical reasons: i) the maximum urea concentrations attainable after serial dilution did not completely unfold BSA; ii) high concentrations of urea, approaching saturation can precipitate easily and block the needle of the syringe pump. Unfolding curves of various BSA:palmitate preparations obtained with fixed volume and serial addition methods are depicted in Figure 4A and 4B respectively. BSA unfolding curves at highpalmitate concentrations display at least one intermediate in the unfolding pathway, as seen from the deviation from a simple two-state unfolding curve. This intermediate is attributed to the early unfolding of the less stable C-terminal part of the protein. Fatty acid molecules partially stabilise the intermediate (Ahmad and Qasim, 1995), increasing the mid-point of urea induced unfolding.

Not all proteins will follow the ideal two-state model of unfolding and simple yet reliable high-throughput data processing protocols will be required for indexing the relative stability of related proteins. Three simple numerical methods were used to quantify the stability of the different BSA preparations: i) the unfolding datasets were fitted to a two-state transition model, calculating an approximate estimate for a global $C_{1 / 2}$-value; ii) the areas below the unfolding curves were calculated using the trapezoidal rule; iii) the denaturant concentration at $60 \%$ or $70 \%$ unfolding was calculated using a linear equation defined by the two data points either side of the arbitrary set value. Figure 5A and 5B depicts the stability estimates using these techniques with urea and $\mathrm{GdnHCl}$ induced unfolding of BSA respectively. Although these are crude estimates of stability, they are still functional and effective at identifying fatty-acid induced stabilisation. All three numerical methods can confidently determine the order of BSA stabilities present at four palmitate 
concentrations. Interestingly, the fluorescence unfolding curves for BSA in GdnHCl in microtiter plates (Figure 4B) can also detect the presence of intermediate states that occur as three palmitate molecules bind to each BSA molecule, ie. at high palmitate concentrations. The unfolding intermediates in this and previously reported urea induced unfolding curves (Ahmad and Qasim, 1995) are less prominent than for the $\mathrm{GdnHCl}$ induced unfolding curves. This demonstrates microtiter plate protein unfolding as a useful high-throughput proteomics method for elucidating the ligandbinding aspects of protein function that alter protein stability.

\section{Conclusions}

We have developed and characterised an affordable high-throughput screening method for the direct measurement of the stability of proteins in a commercially available microplate reader. The method generates unfolding curves in microwells by autotitration of denaturant and measuring the resulting changes in tryptophan fluorescence. The $C_{1 / 2}$ estimate is the most reliable stability parameter obtained with both methods and is directly related to thermodynamic stability $\Delta G_{\mathrm{H}_{2} \mathrm{O}}$ when substituted with [D] in [Eq. (4)]. Analysis of mutant stability requires estimations of $\Delta \Delta G$ values, calculated by substituting [D] with $\Delta C_{1 / 2}$ values in [Eq. (4)] (Kellis, Jr. et al., 1989). This approach assumes that $m_{\mathrm{G}}$-values remain the same for all variants of a protein. The serial addition method is useful when screening for changes in $C_{1 / 2}$ values of greater than $0.15 \mathrm{M}$. Combined with a suitable numerical data analysis technique it can be a powerful tool for screening mutant biocatalyst libraries for improved stability. The fixed volume method produces more reliable datasets and 
parameter determination, but requires more protein, plates and solutions for the unfolding analysis. This method is useful for a more accurate characterisation of either small numbers of rationally designed mutants or a secondary screen, confirming hits from the higher throughput serial-addition screen for protein stability.

Though not all proteins are amenable to fluorescence monitored unfolding, this application can, at least in theory, be extended to absolute extinction coefficient and circular-dichroism based detection. These detection methods also allow for the screening of protein targets for which high-throughput activity screens are difficult to develop. The high-throughput determination of stability in microtiter plates could be applied to the fields of directed evolution, proteomics and therapeutic protein formulation, to produce enzymes stable under process conditions, to characterise functions related to stability changes upon ligand binding, or to ascertain optimal combinations of excipients that prolong the shelf-life of biopharmaceuticals.

The equipment used in this study is functional, and may be readily integrated with conventional automation platforms (Nealon et al, 2004), but is not optimised for this type of work. It can be considered a prototype for dedicated instrumentation that would be modified to particularly accommodate the needs of the high-throughput unfolding analysis. Improvements to consider are accurate titration down to $1 \mu \mathrm{l}$ volumes, solution dispensing close to the surface of the solution in each well, minimised evaporation, active temperature control, and multiple syringe pumps for dispensing various stocks in parallel. While the data in our studies was transferred to a more amenable software package for analysis, flexible software for the integrated data collection, unfolding analysis and stability indexing would also be preferable. All of these modifications are within the reach of current instrumentation technology. As the high-throughput expression and purification of proteins in microwells 
is now fully automatable (Edgell et al., 2003), they could be combined easily with the methods described here to produce a fully automated system for the high-throughput determination of protein stability in microwells, suitable for directed evolution. Such an approach would be highly complementary to related research in our laboratory on the automated microscale process evaluation of evolved biocatalyst libraries (Lye et al., 2003) in linked sequences of fermentation (Elmahdi et al., 2003), bioconversion (Doig et al., 2002) and product recovery operations.

\section{Acknowledgements}

The authors would like to acknowledge the UK Biotechnology and Biological Sciences Research Council (BBSRC) and the Association of Commonwealth Universities (ACU) for financial support. The authors would also like to thank the UK Joint Infrastructure Fund (JIF), the Science Research Investment Fund (SRIF) and the Gatsby Charitable Foundation for funds to establish the UCL Centre for Micro Biochemical Engineering. 


\section{Reference}

Ahmad N, Qasim MA. 1995. Fatty-acid-binding to bovine serum-albumin prevents formation of intermediate during denaturation. Eur J Biochem 227:563-565.

Braun P, LaBaer J. 2003. High throughput protein production for functional proteomics. Trends Biotechnol 21:383-388.

Brems DN, Cass R, Stellwagen E. 1982. Conformational transitions of frog-heart ferricytochrome $c$. Biochemistry 21:1488-1493.

Brodersen R, Honore B, Andersen S. 1988. Palmitate binding to serum-albumin, measured by rate of dialysis. Eur J Biochem 174:45-50.

Burton SG, Cowan DA, Woodley JM. 2002. The search for the ideal biocatalyst. Nature Biotechnology 20:37-45.

Cueto M, Dorta MJ, Munguia O, Llabres M. 2003. New approach to stability assessment of protein solution formulations by differential scanning calorimetry. Int J Pharm 252:159-166.

Dalby PA. 2003. Optimising enzyme function by directed evolution. Curr Opin Struct Biol 13:500-505.

Demirjian DC, Moris-Varas F, Cassidy CS. 2001. Enzymes from extremophiles. Curr 
Opin Chem Biol 5:144-151.

Dobson CM. 1999. Protein misfolding, evolution and disease. Trends Biochem Sci 24:329-332.

Doig SD, Pickering SCR, Lye GJ, Woodley JM. 2002. The use of microscale processing technologies for quantification of biocatalytic Baeyer-Villiger oxidation kinetics. Biotechnology and Bioengineering 80:42-49.

Dyson HJ, Wright PE. 2002. Coupling of folding and binding for unstructured proteins. Curr Opin Struct Biol 12:54-60.

Edgell MH, Sims DA, Pielak GJ, Yi F. 2003. High-precision, high-throughput stability determinations facilitated by robotics and a semiautomated titrating fluorometer. Biochemistry 42:7587-7593.

Elmahdi I, Baganz F, Dixon K, Harrop T, Sugden D, Lye GJ. 2003. pH control in microwell fermentations of $S$. erythraea CA340: influence on biomass growth kinetics and erythromycin biosynthesis. Biochemical Engineering Journal 16:299-310.

Ghaemmaghami S, Fitzgerald MC, Oas TG. 2000. A quantitative, high-throughput screen for protein stability. Proc Natl Acad Sci U S A 97:8296-8301.

Giver L, Gershenson A, Freskgard PO, Arnold FH. 1998. Directed evolution of a 
thermostable esterase. Proc Natl Acad Sci U S A 95:12809-12813.

Hamilton JA, Era S, Bhamidipati SP, Reed RG. 1991. Locations of the 3 primary binding-sites for long-chain fatty acids on bovine serum-albumin. Proc Natl Acad Sci U S A 88:2051-2054.

Heinemann U, Illing G, Oschkinat H. 2001. High-throughput three-dimensional protein structure determination. Curr Opin Biotechnol 12:348-354.

Kellis JT, Jr., Nyberg K, Fersht AR. 1989. Energetics of complementary side-chain packing in a protein hydrophobic core. Biochemistry 28:4914-4922.

Knapp JA, Pace CN. 1974. Guanidine hydrochloride and acid denaturation of horse, cow, and Candida krusei cytochromes $c$. Biochemistry 13:1289-1294.

Kurtzman AL, Govindarajan S, Vahle K, Jones JT, Heinrichs V, Patten PA. 2001. Advances in directed protein evolution by recursive genetic recombination: applications to therapeutic proteins. Curr Opin Biotechnol 12:361-370.

Lakowicz JR. 1999. Principles of fluorescence spectroscopy. New York:Kluwer Academic/Plenum.

Lye GJ, Ayazi-Shamlou P, Baganz F, Dalby PA, Woodley JM. 2003. Accelerated design of bioconversion processes using automated microscale processing techniques. Trends Biotechnol 21:29-37. 
Margoliash E, Frohwirth N. 1959. Spectrum of horse-heart cytochrome $c$. Biochem J $71: 570-572$.

Nealon AJ, Willson KE, Pickering SCR, Clayton TM, O'Kennedy R, TitchenerHooker NJ, Lye GJ. 2004. Use of operating windows in the assessment of integrated robotic systems for the measurement of bioprocess kinetics. Biotechnol Prog submitted.

Pace CN. 1986. Determination and analysis of urea and guanidine hydrochloride denaturation curves. Methods Enzymol 131:266-280.

Pace CN, Vajdos F, Fee L, Grimsley G, Gray T. 1995. How to measure and predict the molar absorption-coefficient of a protein. Protein Sci 4:2411-2423.

Page MJ, Amess B, Rohlff C, Stubberfield C, Parekh R. 1999. Proteomics: a major new technology for the drug discovery process. Drug Discovery Today 4:5562.

Reed RG. 1986. Location of long-chain fatty acid-binding sites of bovine serumalbumin by affinity labeling. J Biol Chem 261:5619-5624.

Remmele RL, Jr., Nightlinger NS, Srinivasan S, Gombotz WR. 1998. Interleukin-1 receptor (IL-1R) liquid formulation development using differential scanning calorimetry. Pharm Res 15:200-208.

Santoro MM, Bolen DW. 1988. Unfolding free-energy changes determined by the 
linear extrapolation method .1. Unfolding of phenylmethanesulfonyl $\alpha$ chymotrypsin using different denaturants. Biochemistry 27:8063-8068.

Scheich C, Niesen FH, Seckler R, Bussow K. 2004. An automated in vitro protein folding screen applied to a human dynactin subunit. Protein Sci 13:370-380.

Schmid A, Dordick JS, Hauer B, Kiener A, Wubbolts M, Witholt B. 2001. Industrial biocatalysis today and tomorrow. Nature (London, UK) 409:258-268.

Schmidt-Dannert C, Arnold FH. 1999. Directed evolution of industrial enzymes. Trends Biotechnol 17:135-136.

Schwehm JM, Stites WE. 1998. Application of automated methods for determination of protein conformational stability. Methods Enzymol 295:150-170.

Vazquez-Duhalt R. 1999. Cytochrome $c$ as a biocatalyst. J Mol Catal B: Enzym 7:241-249.

Weber PC, Salemme FR. 2003. Applications of calorimetric methods to drug discovery and the study of protein interactions. Curr Opin Struct Biol 13:115121.

Won CM, Molnar TE, McKean RE, Spenlehauer GA. 1998. Stabilizers against heatinduced aggregation of RPR 114849, an acidic fibroblast growth factor (aFGF). Int J Pharm 167:25-36. 
Xu Y, Mayne L, Englander SW. 1998. Evidence for an unfolding and refolding pathway in cytochrome $c$. Nat Struct Biol 5:774-778.

Yarmush ML, Jayaraman A. 2002. Advances in proteomic technologies. Annu Rev Biomed Eng 4:349-373.

Yonetani T. 1965. Studies on cytochrome $c$ peroxidase. II. Stoichiometry between enzyme, $\mathrm{H}_{2} \mathrm{O}_{2}$, and ferrocytochrome $c$ and enzymic determination of extinction coefficients of cytochrome $c$. J Biol Chem 240:4509-4514. 


\section{Tables}

Table I. Summary of stability data for oxidized cytochrome $c$ (cyt $c$ ) from bovine and equine heart.

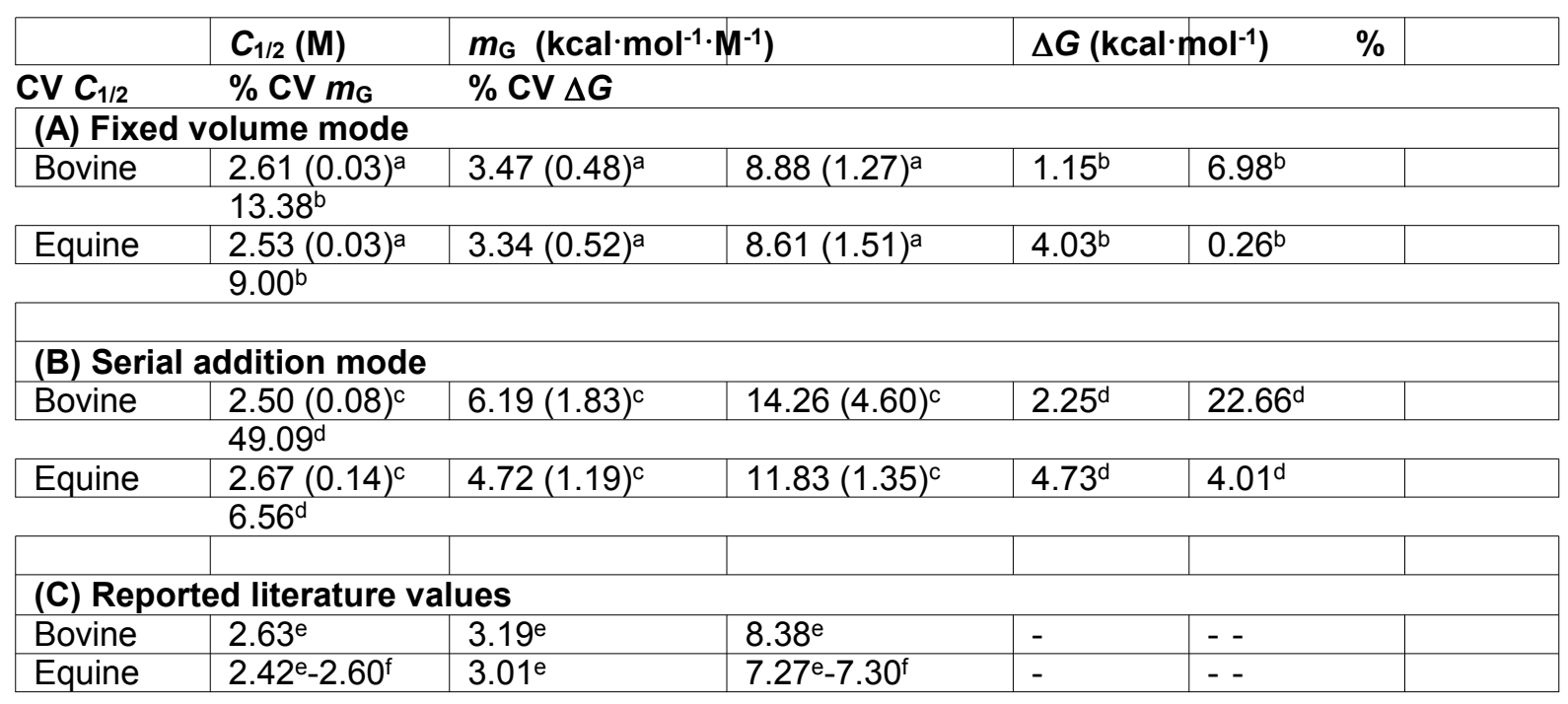

a Standard deviations of the sample $(\sigma)$ calculated from three datasets.

b Percentage coefficient of variation $(\sigma / \bar{x} \times 100)$ calculated from three individual estimates.

c Standard deviations of the population calculated from nine datasets.

d Percentage coefficient of variation $(\sigma / \bar{x} \times 100)$ calculated from twenty-seven datasets.

e (Knapp and Pace, 1974)

f (Brems et al., 1982) 


\section{List of Figures}

Figure 1. The effect of dilution on bovine serum albumin (BSA) fluorescence.

Volumes of $50 \mu \mathrm{l}$ of different concentrations of BSA solutions $(\bullet) 10 \mu{\mathrm{g} \cdot \mathrm{ml}^{-1}}^{-1}$ (o) 30

$\mu \mathrm{g} \cdot \mathrm{ml}^{-1},(\diamond) 60 \mu \mathrm{g} \cdot \mathrm{ml}^{-1},(\nabla) 100 \mu \mathrm{g} \cdot \mathrm{ml}^{-1},(\boldsymbol{\square}) 300 \mu \mathrm{g} \cdot \mathrm{ml}^{-1},(\square) 600 \mu \mathrm{g} \cdot \mathrm{ml}^{-1}$ and $(\bullet)$

$1000 \mu \mathrm{g} \cdot \mathrm{ml}^{-1}$ were pipetted into separate wells of a 96 -well plate. Seven consecutive additions of $25 \mu 1$ were implemented, up to a total volume of $225 \mu 1$, a total dilution of 4.5-fold. All experiments were performed in duplicate wells. (A) shows all data. (B) shows data for only $(\bullet) 10 \mu \mathrm{g} \cdot \mathrm{ml}^{-1}$, (o) $30 \mu \mathrm{g} \cdot \mathrm{ml}^{-1}$, (•) $60 \mu \mathrm{g} \cdot \mathrm{ml}^{-1}$, for greater clarity.

Figure 2. Accuracy of multiple liquid dispensing by the syringe pump into microtiter plates for various volume additions. Linear regressions (-) were fitted to the two sets of data. The slope, $\alpha$, represents the accuracy and the standard error is a measure of the precision. For small injected volumes (o): $\alpha=1.012 \pm 0.007 \mathrm{~g} \cdot \mathrm{cm}^{-3}$; and for large volumes $(\bullet)$ : $\alpha=0.995 \pm 0.001 \mathrm{~g} \cdot \mathrm{cm}^{-3}$.

Figure 3. Conformational transition curves for oxidized cytochrome $c$ (cyt $c$ ) with GdnHCl. (A) Fixed volume method: $20 \mu \mathrm{g}(\bullet)$ bovine heart and (o) horse heart cyt $c$ were loaded per well. Wells were filled to a final volume of $215 \mu \mathrm{l}$ with combinations of $100 \mathrm{mM}$ TrisHCl buffer, $\mathrm{pH} 7.0$ containing $0 \mathrm{M}$ and $6.5 \mathrm{M} \mathrm{GdnHCl}$ to give the correct final denaturant concentration. Each concentration was measured in triplicate. Both data sets show curve fits as described in Materials and Methods. (B) Serial addition method: Multiple replicates of conformational transition curves for 
horse heart cyt $c$. Each curve represents data obtained from a single well in which 20 $\mu \mathrm{g}$ cyt $c$ was loaded. Wells were filled in small increments with the appropriate volume of $100 \mathrm{mM}$ Tris, $\mathrm{pH} 7.0$ containing $6.5 \mathrm{M} \mathrm{GdnHCl}$ to give the correct final denaturant concentration. Plates were agitated for $30 \mathrm{~s}$ followed by an equilibration period of $15 \mathrm{~min}$ before each measurement. One of the plots $(\bullet)$ represents data collected from an edge well. The curve fits are shown for the edge-well plot (•), and for a typical example of the remaining non-edge well plots.

Figure 4. Conformational transition curves for bovine serum albumin (BSA). (A) Fixed volume method: $40 \mu \mathrm{g}$ of BSA stabilised with palmitic acid at molar ratios of

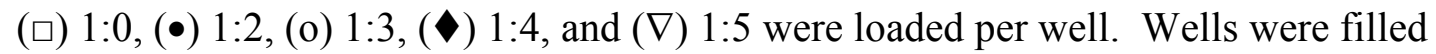
to a final volume of $280 \mu \mathrm{l}$ with combinations of $60 \mathrm{mM}$ sodium phosphate buffer, $\mathrm{pH}$ 7.0 containing $0 \mathrm{M}$ and $9.0 \mathrm{M}$ urea to give the correct final denaturant concentration. Each sample was measured in triplicate. (B) Serial addition method: $50 \mu$ of a 0.8 $\mathrm{mg} / \mathrm{mL}$ BSA solution stabilised with palmitic acid at molar ratios of $(\bullet)$ 1:0, (o) 1:3, $(\checkmark)$ 1:5, ( $\nabla)$ 1:6 and buffered with $60 \mathrm{mM}$ sodium phosphate, $\mathrm{pH} 7.0$, were loaded per well. Unfolding was induced by titrating small volumes of a $5.5 \mathrm{M} \mathrm{GdnHCl} \mathrm{stock}$ solution buffered with $60 \mathrm{mM}$ sodium phosphate, $\mathrm{pH} 7.0$ into each well. Plates were agitated for $30 \mathrm{~s}$ followed by an equilibration period of $15 \mathrm{~min}$ before each measurement. Each plot represents data for an unfolding curve obtained from a single well. Data points are interpolated for the highest BSA:palmitate molar ratio $(\nabla)$, to demonstrate the deviation from a two-state transition.

Figure 5. Stability indexing for palmitate stabilised bovine serum albumin (BSA) 
using different numerical methods. Each dataset in Figure 4 was fit to the two-state unfolding equations 1-4, described in Materials and Methods, even for those cases that clearly deviate from two-state in Figure 4B. Linear increases in stability are represented by values calculated from: $(\bullet)$ denaturant concentration at apparent midpoint of unfolding; ( $)$ denaturant concentration at $60 \%$ unfolding; (o) denaturant concentration at $70 \%$ unfolding; $(\diamond)$ total area under unfolding curve. (A) Data obtained using fixed volume method and urea. (B) Data obtained using serial addition method and GdnHCl. 